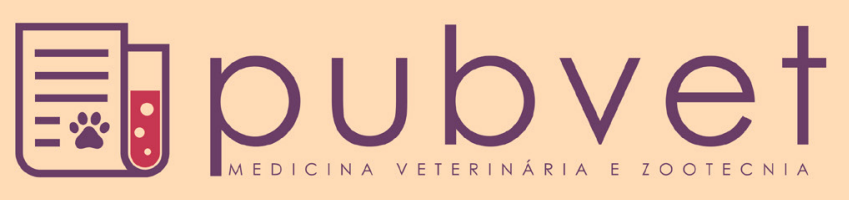

HTTP://DX.DOI.ORG/10.22256/PUBVET.V11N8.781-788

\title{
Estado físico e risco anestésico em cães e gatos: Revisão
}

\author{
Nhirneyla Marques Rodrigues ${ }^{1 *}$, Ana Maria Quessada ${ }^{2}$, Aeyphanny Carlandy Moraes ${ }^{3}$, \\ Sávio Soares Barbosa Dantas ${ }^{4}$, Karina de Kássia da Silva Sales ${ }^{5}$
}

${ }^{I}$ Mestre em Ciência Animal da Universidade Federal do Piaui, Teresina, Piaui, Brasil-nhirneyla@hotmail.com*Autor para correspondência ${ }^{2}$ Professora na área de Ciência Animal da Universidade Paranaense - UNIPAR, Umuarama, Paraná, Brasil-quessadavet@gmail.com ${ }^{3}$ Residente Multiprofissional em Medicina Veterinária - Area: Anestesiologia Veterinária. Universidade de Brasília, Brasília, Distrito Federal, Brasil-phanny_moraes@hotmail.com

${ }^{4}$ Residente Multiprofissional em Medicina Veterinária - Área: Patologia Clínica. Universidade Federal do Piauí, Teresina Piauí, Brasil savioveterinario@hotmail.com

${ }^{5}$ Médica Veterinária, formada pela Universidade Federal do Piauí. Especialista em Clínica e Cirurgia de Cães e Gatos - Qualittas, Teresina, Piaui,Brasil_carinaksales@gmail.com

RESUMO. Grande valia é dada à anestesia desde a sua descoberta. Fato que revolucionou diversas áreas médicas, incluindo a Medicina Veterinária. Para que a anestesia possa ser conduzida de modo adequado e satisfatório, exige-se uma avaliação prévia do paciente a ser submetido à cirurgia, como revisão ao histórico clínico, exame físico, exames complementares e por fim, uma avaliação de risco e estado físico. Sendo importante para direcionar o paciente para um protocolo anestésico condizente ao seu estado de saúde, bem como predizer o prognóstico do mesmo. Esta classificação de risco e estado físico, adotada pela Sociedade Americana de Anestesiologistas (ASA) é aceita e utilizada mundialmente por médicos humanos e veterinários, e amplamente abordada em trabalhos científicos que envolvem a anestesiologia, justificando-se assim, a grande importância da mesma, sendo o objetivo de estudo desta revisão.

Palavras chave: Anestesia, cirurgia, canino, felino

\section{Physical state and anesthetic risk in dogs and cats: Review}

ABSTRACT. Great value has been given to anesthesia since its discovery. A fact that has revolutionized several medical areas, including Veterinary Medicine. In order for anesthesia to be conducted adequately and satisfactorily, a prior evaluation of the patient to be submitted to surgery, such as a review of the clinical history, physical examination, complementary exams and, finally, a risk assessment and physical condition is required. It is important to direct the patient to an anesthetic protocol appropriate to their state of health, as well as to predict the prognosis of the same. This classification of risk and physical status, adopted by the American Society of Anesthesiologists (ASA), is accepted and used worldwide by human and veterinary doctors, and is widely discussed in scientific works involving anesthesiology, justifying its great importance, being the objective of this review.

Keywords: Anesthesia, surgery, canine, feline

\section{Estado físico y riesgo anestésico en perros y gatos: Revisión}

RESUMEN. Gran validez se ha dado a la anestesia desde su descubrimiento. Hecho que revolucionó diversas áreas médicas, incluyendo la Medicina Veterinaria. Para que la anestesia pueda ser conducida de manera adecuada y satisfactoria, se requiere una evaluación previa del paciente a ser sometido a la cirugía, como revisión del historial clínico, examen físico, exámenes complementarios y por fin, una evaluación de riesgo y estado físico. Es importante para dirigir al paciente a un protocolo anestésico que concuerde 
con su estado de salud, así como predecir el pronóstico del mismo. Esta clasificación de riesgo y estado físico, adoptada por la Sociedad Americana de Anestesiólogos (ASA) es aceptada y utilizada mundialmente por médicos humanos y veterinarios, y ampliamente abordada en trabajos científicos que involucran la anestesiología, justificándose así, la gran importancia de la misma, siendo el objetivo de estudio de esta revisión.

Palabras clave: anestesia, canino, cirugía, felino

\section{Introdução}

Há muito tempo, a cirurgia era considerada como algo impiedoso. Assim, um grande salto foi dado ao se descobrir a anestesia, que é tida até hoje como uma das invenções mais valorizadas e importantes para a humanidade (Santos et al., 2015). Cirurgia e anestesia, que passaram então a ser modalidades clínicas indissociáveis, foram utilizadas primariamente em seres humanos, por profissionais da área médica, alquimistas e dentistas, que à época, usavam técnicas rudimentares em anestesia de modo a tentar minimizar a dor produzida em procedimentos cirúrgicos (Aguiar, 2002).

O primeiro profissional a utilizar a anestesia com propósitos cirúrgicos foi o médico Crawford Williamson Long no ano de 1842 . No entanto, só teve seus casos publicados apenas em 1849. Deste modo, o dentista William Thomas Green Morton, que foi responsável pela realização da primeira cirurgia sem dor, em demonstração pública no ano de 1846, levou o total crédito da descoberta. Horace Wells, dentista, também foi um importante personagem nesta descoberta. No entanto, falhou publicamente em sua demonstração anestésica que data de 1845 (Santos et al., 2015).

As substâncias químicas inicialmente utilizadas como anestésicos foram o éter etílico e óxido nitroso (Santos et al., 2015). Junto à descoberta da anestesia, a aplicação de métodos antissépticos, foi outro marco histórico decisivo para que a cirurgia atingisse o seu presente estágio de desenvolvimento (McCurnin and Jones, 2007). Desta maneira, a preparação prévia do paciente e da equipe cirúrgica, tende a diminuir os riscos de infecção, sendo utilizados para tal fim: antissépticos adequados e barreiras estéreis entre as superfícies contaminadas e a incisão, de modo a reduzir a contaminação bacteriana da ferida (Fries, 2007).

$\mathrm{Na}$ Medicina Veterinária, os passos percorridos foram um pouco mais lentos, sendo que o uso inicial de técnicas anestésicas se deu apenas a partir do final do século XIX, por Edward Mayhew, utilizando éter em seus experimentos com cães e gatos (Aguiar, 2002). O primeiro médico veterinário a utilizar éter e clorofórmio de forma rotineira em procedimentos cirúrgicos foi George H. Dadd, no ano de 1852 e no Brasil, os primeiros relatos sobre anestesiologia veterinária se deram apenas a partir da década de 1940 (Aguiar, 2002). Com o decorrer dos anos, muitas técnicas anestésicas e cirúrgicas na Medicina Veterinária foram incrementadas variando conforme o estado geral do paciente, idade, presença de dor e doenças associadas (Fantoni et al., 2002).

Muitas vezes, a garantia de uma cirurgia com bons resultados pode ser determinada por uma avaliação minuciosa no período pré-operatório, permitindo estabilizar ou mesmo preparar adequadamente o paciente, minimizando-se o risco e o potencial de complicações no período pós-operatório (Fries, 2007). A esta conduta, se dá o nome de avaliação pré-anestésica e é por meio dela que se obtêm dados referentes ao histórico clínico do paciente, exames complementares, permitindo, ainda, estimar o risco anestésicocirúrgico do paciente, planejar a anestesia e aplicar cuidados perioperatórios essenciais (Futema, 2002).

A avaliação pré-anestésica deve ser considerada parte integrante da técnica de anestesia e visa a correção dos desequilíbrios orgânicos do paciente antes que o mesmo seja submetido à anestesia, minimizando as possíveis complicações decorrentes destas alterações (Laredo et al., 2001). Uma anestesia realizada com segurança e eficácia resulta, dentre outros fatores, de uma boa preparação e avaliação prévia, devendo ser levadas em consideração várias características individuais de cada paciente, como: histórico clínico, idade, raça, temperamento do animal, exame físico e o tipo de procedimento cirúrgico que será realizado (Bednarski et al., 2011).

Esta avaliação minuciosa, além de fundamental no preparo do paciente, ainda é considerada como ferramenta auxiliar, direcionando a solicitação de exames complementares, evitando exames desnecessários e contribuindo assim com a redução dos custos 
(Issa et al., 2011), mas sem haver perda de dados clínicos importantes (Ferrando et al., 2005). Todavia, há situações emergenciais, no qual o risco de se adiar a cirurgia é considerado maior que o risco anestésico deste momento, devendo, portanto ser realizada a intervenção cirúrgica o mais brevemente (Laredo et al., 2001). Isto exige maior capacitação do profissional envolvido, abrangendo o conhecimento de diversas áreas da veterinária como: cirurgia, anestesiologia, oncologia, cardiologia, neurologia e outras, estando este profissional apto, portanto, a tomar decisões e intervir rapidamente, já que isto pode ser a diferença entre a vida e a morte do paciente.

\section{Revisão de literatura}

Na prática clínica veterinária é de grande valia a obtenção de um histórico minucioso da vida do paciente e realização de exame físico completo, sendo este, um dos principais métodos de determinação do diagnóstico de diversas enfermidades, evitando ainda, custos desnecessários ao proprietário com exames auxiliares que pouco contribuiria na determinação do diagnóstico definitivo (Birchard and Sherding, 2008). No paciente cirúrgico, esta avaliação é particularmente útil porque pode minimizar complicações anestésicas e cirúrgicas que possam ocorrer, além de permitir a busca por afecções concomitantes e manifestações sistêmicas do problema primário (Fries, 2007).

Deste modo, deve-se, inicialmente, realizar a anamnese do paciente, entrevistando seu tutor acerca do problema clínico que o animal está apresentando e o impacto que o mesmo tem causado no seu estado geral de saúde (Shmon, 2007). Por meio deste histórico podem ser identificadas patologias anteriores, doenças atuais, fatores de risco, saber o que já lhe foi prescrito e se já houve reações adversas a algum medicamento (Bednarski et al., 2011). Em seguida, identifica-se o paciente, de modo a determinar problemas de saúde inerentes à idade, raça e sexo (Shmon, 2007). A idade por si só não é contraindicação para a anestesia; porém, o paciente canino ou felino idoso, pode ter patologias relacionadas à sua idade avançada, havendo diminuição de reservas ou funcionalidade de um órgão. Por isso, neste grupo de pacientes deve ser realizada avaliação préoperatória completa e escolha cuidadosa das técnicas anestésicas e analgésicas. $\mathrm{O}$ monitoramento do mesmo deve ser feito de forma vigilante e cuidadosa, proporcionando-se suporte adequado, melhorando a probabilidade de um resultado positivo para o paciente idoso (Hughes, 2008).

Nos pacientes muito jovens, há um maior risco de ocorrência de hipotermia, hipoglicemia e diminuição do metabolismo de fármacos (Bednarski et al., 2011). Portanto, nestes pacientes, são necessários condutas e fármacos que contornem tais situações clínicas. Quanto à raça, características próprias devem ser consideradas já que determinadas raças apresentam incidência maior de condições que afetam a conduta anestésica e cirúrgica como, por exemplo, raças braquicefálicas que necessitam de exame mais criterioso nas vias respiratórias superiores (Shmon, 2007).

No exame físico do paciente, realizado após obtenção da anamnese e histórico clínico, o profissional deve fazer uso dos sentidos: visão, audição, olfato e tato para procurar indícios de patologia (Ortenzi, 2006). Nada substitui estas ferramentas próprias do examinador. Deve-se verificar o peso corporal atual do paciente e avaliar o seu escore corporal, já que muitas vezes, ele pode ser indicador de prognóstico de algumas enfermidades como, por exemplo, na doença renal crônica, no qual a caquexia está relacionada à maior mortalidade (Nobre et al., 2010). Já em situação contrária, a obesidade aumenta o risco cirúrgico por dificultar a ventilação, em decorrência do acúmulo de gordura intratorácica (Shmon, 2007). Ainda no exame físico, atenção deve ser dada aos sistemas cardiovascular e respiratório, logo, os sinais vitais devem ser aferidos: frequência cardíaca (em batimentos por minuto), pulso, frequência respiratória (em movimentos por minuto) e temperatura corporal (em graus Celsius) (Birchard and Sherding, 2008).

Devem ser considerados ainda os seguintes parâmetros: hidratação, geralmente verificada pelo turgor cutâneo, presença ou não de retração do globo ocular ou protrusão bilateral da terceira pálpebra (sinais típicos de um animal desidratado). A coloração das mucosas deve ser verificada, sendo a conjuntiva e oral as mais acessíveis, investigando-se alterações como: hiperemia, palidez, icterícia e outras (Birchard and Sherding, 2008). Todas as alterações referentes à hidratação, temperatura, coloração das mucosas e tempo de preenchimento capilar devem ser verificados para se evidenciar presença de anemia e anormalidades circulatórias (Shmon, 2007). 
Após o exame geral, deve-se proceder à avaliação da área afetada e dos órgãos adjacentes, embasando assim, a descrição do estado físico do animal e seu risco anestésico-cirúrgico (Fries, 2007). Quanto aos exames complementares no pré-operatório, os mesmos devem ser solicitados de modo seletivo, em função do exame físico minucioso, com o propósito básico de guiar e otimizar o cuidado perioperatório, levando em consideração as informações obtidas no prontuário do paciente, histórico clínico, exame físico, tipo e porte do procedimento cirúrgico (Mathias et al., 2006) (Tabela 1).

Tabela 1. Sugestão de exames complementares pré-anestésicos, de acordo com a categoria de estado físico Sociedade Americana de Anestesiologistas (ASA) e idade em cães e gatos.

\begin{tabular}{|c|c|c|c|}
\hline Estado Físico ASA & Até 6 meses & 6 meses a 6 anos & Mais de 6 anos \\
\hline I e II & $\begin{array}{l}\text { Hematócrito, } \\
\text { glicemia }\end{array}$ & $\begin{array}{l}\text { Hematócrito, proteína, função } \\
\text { renal }\end{array}$ & $\begin{array}{l}\text { Hematócrito, proteína, função } \\
\text { renal, ECG, urinálise }\end{array}$ \\
\hline II & $\begin{array}{l}\text { Hematócrito, proteína, } \\
\text { glicemia, função renal, pH, } \\
\text { HCO3 e gases sanguíneos, } \\
\text { urinálise }\end{array}$ & $\begin{array}{l}\text { Hemograma, ECG, glicemia, } \\
\text { função renal, função hepática, } \\
\text { pH, HCO3 e gases sanguíneos, } \\
\text { urinálise }\end{array}$ & $\begin{array}{l}\text { Hemograma, glicemia, ECG, } \\
\text { função renal, função hepática, } \\
\text { eletrólitos (Na+, K+, Ca+2), } \\
\text { pH, HCO3 e gases sanguíneos, } \\
\text { urinálise }\end{array}$ \\
\hline IV e $\mathrm{V}$ & $\begin{array}{l}\text { Hemograma, ECG, glicemia, } \\
\text { função renal, função hepática, } \\
\text { eletrólitos }(\mathrm{Na}+\mathrm{K}+, \mathrm{Ca}+2) \text {, } \\
\text { pH, HCO3 e gases sanguíneos, } \\
\text { urinálise }\end{array}$ & $\begin{array}{l}\text { Hemograma, ECG, glicemia, } \\
\text { função renal, função hepática, } \\
\text { eletrólitos }(\mathrm{Na}+\mathrm{K}+, \mathrm{Ca}+2) \text {, } \\
\text { pH, HCO3 e gases sanguíneos, } \\
\text { urinálise }\end{array}$ & $\begin{array}{l}\text { Hemograma, ECG, glicemia, } \\
\text { função renal, função hepática, } \\
\text { eletrólitos (Na+, K+, Ca+2), } \\
\text { pH, } \mathrm{HCO} 3 \text { e gases sanguíneos, } \\
\text { urinálise }\end{array}$ \\
\hline E: (Emergência) & \multicolumn{3}{|c|}{$\begin{array}{l}\text { Se o procedimento cirúrgico for de duração inferior a } 60 \text { minutos: VG, PT e urinálise. } \\
\text { Se a cirurgia for realizada em tempo superior a } 60 \text { minutos e o paciente com mais de } 7 \text { anos de idade: } \\
\text { os testes dependem das instalações disponíveis. }\end{array}$} \\
\hline \multicolumn{4}{|c|}{$\begin{array}{l}\text { Observações: } \\
\text { A gasometria (pH, HCO3 e gases sanguíneos) poderá ser requisitada pelo anestesista apenas na pré-anestesia; } \\
\text { A urinálises poderá ser requisitada apenas nos casos de sintomatologia que envolva o trato geniturinário; } \\
\text { Faz-se necessária avaliação e cálcio nos animais portadores de neoplasias. }\end{array}$} \\
\hline
\end{tabular}

Os pacientes hígidos, que serão submetidos à cirurgia eletiva, necessitam de menos exames complementares, diferentemente de pacientes que necessitam de intervenções cirúrgicas mais complexas, uma vez que há maior probabilidade destas anormalidades afetarem o resultado final da cirurgia (Shmon, 2007).

Vale ressaltar que condições emergenciais, que possuem maior risco de complicação e consequentemente de óbito, podem limitar a avaliação e estabilização pré-operatórias possíveis, daí a necessidade de intervir rapidamente (Shmon, 2007). Após finalizar a análise do prontuário clínico do paciente, exame físico e obtidos os resultados de exames complementares solicitados, pode-se atribuir a categoria de estado físico a qual o animal pertence, conforme estabelecido pela Sociedade Americana de Anestesiologistas.

Historicamente, em 1941, o pesquisador Saklad elaborou uma classificação de estado físico, considerando-se a mortalidade secundária à anestesia e as condições clínicas pré-operatórias, que foi adotada posteriormente pela ASA logo após adição da quinta categoria e hoje é empregada quase universalmente (Ortenzi, 2006), tanto por médicos humanos como na Medicina Veterinária, tendo o mesmo objetivo, que é normatizar as condutas da avaliação préanestésica, as abordagens a serem realizadas, que incluem: revisão do prontuário, exames físicos e laboratoriais, histórico de anestesias anteriores, medicações em uso e classificação quanto ao estado físico (Owens et al., 1978, Novaes, 2006). Esta classificação ASA é comumente utilizada na avaliação pré-anestésica de seres humanos e animais, sendo um parâmetro bastante avaliado em pesquisas nas áreas de cirurgia e anestesiologia (Barreiro and Garat, 1992, Cicarelli et al., 1998, Redondo et al., 1998, Chan and Auler Júnior, $\underline{2002}$, Schwartzman et al., 2011). A classificação de estado físico e risco anestésico qualifica o paciente cirúrgico de I a VI, variando conforme as características observadas ( $\underline{\text { ASA, 2013): }}$

ASA I - aparentemente hígido, sem alterações orgânicas;

ASA II - apresentando doença sistêmica de grau leve; 
ASA III - doença sistêmica moderada;

ASA IV - presença de doença sistêmica grave;

ASA V - pacientes moribundos, sem expectativa de sobrevivência com ou sem cirurgia nas próximas 24 horas;

ASA VI - paciente com morte cerebral cujos órgãos estão sendo removidos para fins doação.

Todas estas classificações podem ser qualificadas como condição de emergência " $E$ " (ASA, 2013), que são casos em que há alta probabilidade de consequências imediatas de risco de vida, ou que necessitam de cuidados e intervenções imediatas (Fragata and Santos, 2008). Como exemplos de cada grupo de risco, são citados: ASA I - animais submetidos a procedimentos eletivos como orquiectomia, ovariossalpingohisterectomia e conchectomia; ASA II - pacientes neonatos e geriátricos, gestantes, obesos, cardiopatas compensados, fraturas simples, lacerações de pele e os que possuem infecções localizadas; ASA III - casos de animais anêmicos, caquéticos, que apresentem sopro cardíaco, desidratação moderada, hipovolemia, hérnia diafragmática, pneumonia, pneumotórax, trauma torácico discreto e casos de fraturas complicadas; ASA IV - casos de: choque, toxemia, uremia, hipovolemia, desidratação grave, doenças cardíacas e renais descompensadas, anemia grave, síndrome torção-dilatação gástrica e piometra; ASA V - exemplificados por casos de: traumas cranianos, choque e falência de múltiplos órgãos (Futema, 2002, Shmon, 2007).

Em pacientes humanos há o grupo denominado ASA VI, referente aos pacientes doadores de órgãos (Novaes, 2006) e (ASA, 2013), prática ainda incomum na Medicina Veterinária.

De um modo geral, a avaliação pré-operatória, na qual a classificação ASA de estado físico está inserida, mostra-se como importante ferramenta de segurança ao paciente cirúrgico, visando à redução de intercorrências intra e pós-operatórias, evitando-se assim, desfechos desfavoráveis (Schwartzman et al., 2011). São relatados em seres humanos erros e acidentes no bloco operatório, que poderiam ser evitados com a prévia avaliação do paciente (Fragata, 2010). Embora não sejam registrados em Medicina Veterinária, estes mesmos erros provavelmente ocorrem. Portanto, a implantação da avaliação pré-anestésica de forma rotineira, pode contribuir na eficiência dos serviços ofertados e também na melhoria da formação dos anestesiologistas (Bisinotto et al., 2007).
Quando realizada de modo minucioso, a avaliação pré-operatória permite diminuir os custos quanto aos exames solicitados, sem que haja perda da qualidade e informações clinicamente relevantes (Ferrando et al., 2005, Issa et al., 2011) e possibilita diminuição no tempo de permanência hospitalar (Mendes et al., 2005). Consequentemente, os pacientes cujo risco préoperatório é baixo (ASA I ou II) são os principais contribuintes para a redução do número de exames pré-operatórios e custos desnecessários (Ferrando et al., 2005). Assim, confirma-se a grande importância da classificação ASA pré-operatória, sendo que a mesma deve ser incluída na descrição do estado físico pré-anestésica do animal, uma vez que permite estimar os riscos de possíveis complicações anestésicas e cirúrgicas, bem como fornecer o prognóstico do paciente (Luz et al., 2012, Fossum, 2014) que é considerado como excelente, bom, satisfatório, reservado e grave, para as categorias ASA I, II, III, IV e V respectivamente e, prognóstico variável para pacientes emergenciais (Shmon, 2007).

O tratamento do paciente com base numa avaliação pré-anestésica completa se torna bem mais barato que o tratamento decorrente de complicações inesperadas (Shmon, 2007). Apesar da incontestável utilidade da classificação ASA de estado físico, a mesma não mostra total precisão científica (Owens et al., 1978), ou seja, é de fácil aplicação, importante na avaliação pré-anestésica do paciente, contudo, não é preciso o suficiente para garantir que todos os anestesiologistas classifiquem o paciente de forma semelhante. No entanto, a mesma tem se mostrado necessária no fim da avaliação realizada e segundo pesquisas, tem demonstrando estreita relação entre morbidades e mortalidades anestésicas (Ortenzi, 2006). Entende-se por mortalidade, o índice que registra o número de indivíduos que vêm a óbito em determinado intervalo de tempo. $\mathrm{Na}$ anestesiologia humana, que é tida como a área mais segura da medicina, possui baixíssimas taxas de óbito diretamente relacionadas ao risco anestésico (ASA I e II), sendo detectada uma morte para cada 250 a 300 mil anestesias em seus pacientes (Fragata, 2010).

Apesar dos dados supracitados, cabe lembrar que todos os pacientes submetidos à anestesia querem seres humanos ou outros animais, podem apresentar eventuais intercorrências, mesmo que estejam classificados em ASA I ou II (Futema, 2002). Isto pode ser notado com base nos resultados de um estudo realizado em cães, no qual 
houve complicações e mesmo óbito, em pacientes cuja determinação de risco ASA foi inicialmente dada como baixa: ASA I e II (Carareto et al., 2005).

Pesquisas sobre a incidência de óbitos anestésico-cirúrgicos são bem mais comuns na medicina humana, nos quais se obteve maior taxa de mortalidade nas primeiras 24 horas em pacientes idosos, do sexo masculino, classificados em estado físico ASA IV, sendo a maioria das mortes inevitáveis (Cicarelli et al., 1998). Em outro estudo retrospectivo, também referente aos óbitos anestésico-cirúrgicos nas primeiras 24 horas, notou-se que os óbitos foram considerados inevitáveis, cuja incidência maior se deu em pacientes neonatos. Houve predominância de mortes no sexo masculino, pacientes classificados como ASA III ou mais, em cirurgias de emergência, cardíacas ou vasculares (Chan and Auler Júnior, 2002).

De modo semelhante, levantamentos com essa temática são bastante relevantes e necessários, embora ainda em pequeno número na Medicina Veterinária. Em estudo retrospectivo foram analisadas 7.012 fichas anestésicas de caninos e felinos, em um intervalo de tempo que totalizou 11 anos, obtendo-se altos índices de mortalidade para ambas as espécies, no uso de fármacos comprovadamente seguros, sugerindo que este resultado tenha sido em função da maior aplicação destes agentes em pacientes com alterações sistêmicas (ASA III, IV e V) (Corrêa et al., 2009). Estudo sobre risco de óbitos relacionados à anestesia em cães demonstrou que este risco está relacionado ao aumento do grau do estado físico ASA, diminuição do peso corporal, urgência na realização do procedimento cirúrgico e maior duração de tempo do mesmo (Brodbelt et al., 2007). Em levantamento retrospectivo recente (2007-2011) referente a procedimentos anestésicos realizados na Universidade Federal do Rio Grande do Sul, em 3.834 cães e 1.532 gatos, cuja incidência de óbito foi de $0,96 \%$ do total dos procedimentos, sendo $0,55 \%$ para pacientes hígidos e $1,32 \%$ para os doentes, comprovando que a classificação ASA é importante para estabelecer o risco anestésico em procedimentos cirúrgicos (Luz et al., 2012). Quando se compara as taxas de óbitos relacionados à anestesia entre as espécies canina e felina, a última tem apresentado maior risco na maioria dos estudos (Corrêa et al., 2009, Brodbelt, 2009), mas há outros que citam maior incidência de óbitos em cães, especialmente nos doentes (Luz et al., 2012). Maiores índices de morbidade e mortalidade também são observados em casos de procedimentos cirúrgicos extensos, realizados em órgãos vitais ou em casos de emergência (Ortenzi, 2006).

Trabalhos realizados com este tema enriquecem as informações já existentes na literatura e divulgam dados relevantes acerca dos serviços anestésico-cirúrgicos oferecidos. De modo geral, com o decorrer dos anos, é possível observar melhorias na realização de procedimentos anestésicos, havendo também, redução dos riscos de óbito em pequenos animais (Birchard and Sherding, 2008).

\section{Referências bibliográficas}

American Society of Anesthesiologists (ASA). ASA Physical Status Classification System. Disponível em: $<\mathrm{http}$ ://www.asahq.org/Home/ForMembers/Clinical-Information/ASA-PhysicalStatus-Classification-System>. Acesso em 06 fev. 2017.

Aguiar, A. J. A. 2002. História da anestesia. In: Fantoni, D. T. \& Cortopassi, S. R. G. (eds.) Anestesia em cães e gatos. Roca, São Paulo, Brasil.

Barreiro, G. \& Garat, J. 1992. Incidentes críticos em anestesia. Revista Brasileira de Anestesiologia, 42, 357-76.

Bednarski, R., Grimm, K., Harvey, R., Lukasik, V. M., Penn, W. S., Sargent, B. \& Spelts, K. 2011. AAHA anesthesia guidelines for dogs and cats. Journal of the American Animal Hospital Association, 47, 377-385.

Birchard, S. J. \& Sherding, R. G. 2008. Manual Saunders: clínica de pequenos animais, São Paulo.

Bisinotto, F. M. B., Pedrini Júnior, M., Alves, A. A. R. \& Andrade, M. 2007. Implantação do serviço de avaliação pré-anestésica em hospital universitário. Dificuldades e resultados. Revista Brasileira de Anestesiologia, 57, 167176.

Brodbelt, D. 2009. Perioperative mortality in small animal anaesthesia. The Veterinary Journal, 182, 152-161.

Brodbelt, D. C., Pfeiffer, D. U., Young, L. E. \& Wood, J. L. N. 2007. Risk factors for anaesthetic-related death in cats: results from the confidential enquiry into perioperative small animal fatalities (CEPSAF). British Journal of Anaesthesia, 99, 617-623. 
Carareto, R., Rocha, L. S., Guerrero, P. N. H., Sousa, M. G., Nunes, N., Paula, D. P. \& Nishimore, C. T. 2005. Estudo retrospectivo da morbidade e mortalidade associada com anestesia geral inalatória em cães. Semina: Ciência Agrárias, 26, 569-574.

Chan, R. P. C. \& Auler Júnior, J. O. C. 2002. Estudo restrospectivo da incidência de óbitos anestésico-cirúrgicos nas primeiras 24 horas. Revisão de 82.641 anestesias. Revista Brasileira de Anestesiologia, 52, 719-727.

Cicarelli, D. D., Gotardo, A. O. M., Júnior, A., Costa, J. O., Olivetti, G. T. \& Oliveira, F. S. 1998. Incidência de óbitos anestésicocirúrgicos nas primeiras 24 horas. Revisäo de prontuários de 1995 no Hospital das Clínicas da FMUSP. Revista Brasileira de Anestesiologia, 48, 289-94.

Corrêa, A. L., Oleskovicz, A. L. C. N. \& Moraes, A. N. 2009. Índice de mortalidade durante procedimentos anestésicos: estudo retrospectivo (1996-2006). Ciência Rural, 39, 2519-2526.

Fantoni, D. T., Mastrocinque, S., Fantoni, D. T. \& Cottopassi, S. R. 2002. Fisiopatologia e controle da dor. In: Fabntoni, D. T. (ed.) Anestesia em cães e gatos. Roca, São Paulo.

Ferrando, A., Ivaldi, C., Buttiglieri, A., Pagano, E., Bonetto, C., Arione, R., Scaglione, L., Gelormino, E., Merletti, F. \& Ciccone, G. 2005. Guidelines for preoperative assessment: impact on clinical practice and costs. International Journal for Quality in Health Care, 17, 323-329.

Fossum, T. W. 2014. Cirurgia de pequenos animais, 4 edn. Elsevier Brasil, São Paulo.

Fragata, F. S. \& Santos, M. M. 2008. Principais Conceitos em Medicina Veterinária Intensiva. Roca, São Paulo, Brasil.

Fragata, J. I. G. 2010. Erros e acidentes no bloco operatório: revisão do estado da arte. Revista Portuguesa de Saúde Pública, 6, 17-26.

Fries, C. L. 2007. Avaliação e preparação do paciente cirúrgico. In: Slatter, D. (ed.) Manual de cirurgia de pequenos animais. Manole, São Paulo Brasil.

Futema, F. 2002. Avaliação pré-anestésica. Roca, São Paulo, Brasil.

Hughes, J. M. L. 2008. Anaesthesia for the geriatric dog and cat. Irish Veterinary Journal, 61, 380-387.
Issa, M. R. N., Fernandes, M. L., Soares, A. M. \& Isoni, N. F. C. 2011. Avaliação pré-anestésica e redução dos custos do preparo pré-operatório. Revosta Brasileira de Anestesiologia, 6, 60-71.

Laredo, F., Redondo, J., Gómez-Villamandos, R. \& Belda, E. 2001. La preanestesia: analgesia, inmovilización farmacológica, tranquilización y ansiolisis. Consulta de Difusión Veterinaria, 9, 37-50.

Luz, L. C., Muccillo, M. S., van der Laan Fonini, A., Ledur, G. R., Kasper, P. N. \& Contesini, E. A. 2012. Mortalidade em anestesia de cães e gatos: estudoretrospectivo de 5.366 procedimentos anestésicos. Archives of Veterinary Science, 17, 1-5.

Mathias, L. A. S. T., Gozzani, J. L., Rivetti, L. A. \& Guaratini, Á. A. 2006. Exames complementares pré-operatórios: análise crítica. Revista Brasileira de Anestesiologia, 56, 658-668.

McCurnin, D. M. \& Jones, R. L. 2007. Princípios da assepsia cirúrgica. In: Slatter, D. (ed.) Manual de cirurgia de pequenos animais. Manole, São Paulo Brasil.

Mendes, F. F., Mathias, L. A. S. T., Duval Neto, G. F. \& Birck, A. R. 2005. Impacto da implantação de clínica de avaliação préoperatória em indicadores de desempenho. Revista Brasileira de Anestesiologia, 55, 175187.

Nobre, M. C., Vieira, A. B., Santos, M. C. S., Gershony, L. C., Soares, A. M. B. \& Ferreira, A. M. R. 2010. Escore de condição corporal como indicador do prognóstico de gatos com doença renal crônica. Ciência Rural, 40, 335340.

Novaes, M. V. 2006. Avaliação e preparo préoperatório: classificação do estado físico. Serviço de Anestesiologia de Joinville, 1, 1121.

Ortenzi, A. V. 2006. Avaliação pré-anestésica. Organização Sociedade de Anestesiologia do Estado de São Paulo. São Paulo.

Owens, W. D., Felts, J. A. \& Spitznagel Junior, E. L. 1978. ASA physical status classifications: a study of consistency of ratings. Anesthesiology, 49, 239-243.

Redondo, J. I., Gómez Villamandos, R. J., Santisteban Valenzuela, J. M., Ruiz, I., Domínguez Pérez, J. M. \& Ávila, I. 1998. Complicaciones en la anestesia general del perro. Revisión de 265 Casos. Clínica 
Veterinaria de Pequeños Animales, 18, 0087100.

Santos, M. A., Oliveira, M. F., Nunes, P. N., Dias, T. L. L., Marcelino, V. S., Malozze, P. C. \& Tonolli, D. M. 2015. Anestesia: aspectos históricos. UNILUS Ensino e Pesquisa, 12, 120.

Schwartzman, U. P., Batista, K. T., Duarte, L. T. D., Saraiva, R. A. \& Fernandes, M. C. B. C. 2011. Complicações anestésicas em cirurgia plástica e a importância da consulta préanestésica como instrumento de segurança. Revista Brasileira de Cirurgia Plástica, 26, 221-227.
Shmon, C. 2007. Avaliação e preparação do paciente e da equipe cirúrgica. In: Slatter, D. (ed.) Manual de cirurgia de pequenos animais. Manole, São Paulo Brasil.

\section{Article History:}

Received 15 April 2017

Accepted 17 May 17

Available on line 28 June 2017

License information: This is an open-access article distributed under the terms of the Creative Commons Attribution License 4.0, which permits unrestricted use, distribution, and reproduction in any medium, provided the original work is properly cited. 